\title{
Quantitative Compositional Mapping and Multivariate Statistical Analysis: An EDS Approach
}

\author{
S. M. Seddio ${ }^{1}$ and P. K. Carpenter ${ }^{2}$ \\ ${ }^{1}$ Thermo Fisher Scientific, 5225 Verona Rd., Fitchburg, WI, 53711, USA \\ ${ }^{2}$ Dept. of Earth and Planetary Sciences, Washington University, CB1169, Saint Louis, MO, 63130, USA
}

Rock samples typically exhibit textural and chemical features requiring mineral analysis over cm-sized areas using major, minor, and trace elements. This need has driven research in fixed-beam stage mapping using the electron microprobe (EPMA) to collect X-ray maps by wavelength-dispersive X-ray spectroscopy (WDS) [1]. This need has also motivated research to investigate to what extent EPMA results can be reproduced using SEM-based EDS (hereafter, EDS). EDS spot quantitative analysis uses probe current and count time values such as $1-5 \mathrm{nA}$ and $30 \mathrm{~s}$ for major elements and up to $1000 \mathrm{~s}$ for trace elements (EDS typically has higher detection limits than does WDS). These conditions yield a total dose range of 30 - 5000 nA-s. Quantitative EDS map acquisitions may be acquired using a fixed-beam, stage map method but are more commonly done by rastering the electron beam over multiple fields of view of the SEM and montaging these acquisitions together. EDS map acquisitions do not require multiple passes to acquire all EDS data because all fluoresced X-rays are concurrently acquired. Additionally, these acquisitions are hyperspectral images in which an entire EDS spectrum is stored at each pixel, which enables the extraction of qualitative and quantitative elemental maps and phase maps (determined using multivariate statistical analysis [2,3]-MSA) from the same dataset. Typical EDS spectral image acquisitions for the purposes of phases mapping or mapping qualitative elemental distributions use $1-10 \mathrm{nA}$ and $<1$ ms/pixel, representing a dose range of $0.0001-0.01 \mathrm{nA}-\mathrm{sec}$, several orders of magnitude lower than for spot analysis. This dosage metric is useful for evaluating the measurement precision and detection limit for conventional vs. map analysis by using a given X-ray count rate, and reveals that mapping measurements acquired using typical conditions have significantly lower precision and therefore higher detection limits. Quantitative (standardless and standards-based) compositional mapping runs are made at higher probe currents to compensate for shorter dwell times, and calculated detection limit maps can be used for quality control of data. Here, the precision and accuracy dependence on mapping conditions is discussed.

A rock sample (Fig. 1) is used for testing these parameters ( [1] has more sample detail). Spot analyses of core and rim compositions define the Ca-pyroxene zoning by elements $\mathrm{Mg}, \mathrm{Al}, \mathrm{Ti}$, and $\mathrm{Cr}$, summarized in Table 1 of [1]. EDS spectral images were acquired from a 1 -inch round polished section using a $0.72 \times$ $0.54 \mathrm{~mm}^{2}$ map area at $256 \times 192$ pixels using a Thermo Scientific $100 \mathrm{~mm}^{2}$ UltraDry EDS detector and processed using the Pathfinder X-ray microanalysis system. Spectral images were acquired in a dynamically templated manner to avoid acquiring X-ray data from fractures and epoxy [4]. Two map runs were conducted at $15 \mathrm{kV}$ with a run acquired at $0.5 \mathrm{nA}-\mathrm{s}(10 \mathrm{nA}$ and $49 \mathrm{~ms} / \mathrm{pixel})$ and a run at $1.0 \mathrm{nA}-\mathrm{s}(10 \mathrm{nA}$ and $98 \mathrm{~ms} / \mathrm{pixel}$ ). The EDS standardless quantitative mapping for the 0.5 and $1.0 \mathrm{nA}$-s acquisitions exhibit zoning in $\mathrm{Mg}, \mathrm{Al}$, and $\mathrm{Ti}$ (Fig. 1). In both acquisitions, Cr concentrations are below the detection limit.

MSA of EDS spectral images enables the discrimination of phases based on subtle differences in spectral shapes with minimal X-ray counts/pixel. MSA of the $0.5 \mathrm{nA}-\mathrm{s}$ dose EDS spectral images discriminated between the zones of the Ca-pyroxene for $\sim 50 \%$ of the pixels representing Ca-pyroxene. MSA of the 1.0 nA-s dose EDS spectral images successfully discriminated between the zones of the Ca-pyroxene, which were not be discriminated using cluster analysis [1]. The component maps for the mapped area are in 
Figure 2. Future work will include standards-based quantitative mapping on larger areas in order to extract detection limit maps and to overall better match the mapping done in the companion abstract [1].

\section{References}

[1] P Carpenter, Microsc. Microanal. 2018. This Conference.

[2] Keenan et al., Meth. of Multivariate Spectral Analysis. Pat. 6,675,106 B1. 06Jan.2004.

[3] Keenan et al., Apparatus and Sys. for Multivariate Spectral Analysis. Pat. 6,675,106 B1. 06Jan.2004. [4] S Seddio, Microsc. Microanal. 2018. This Conference.

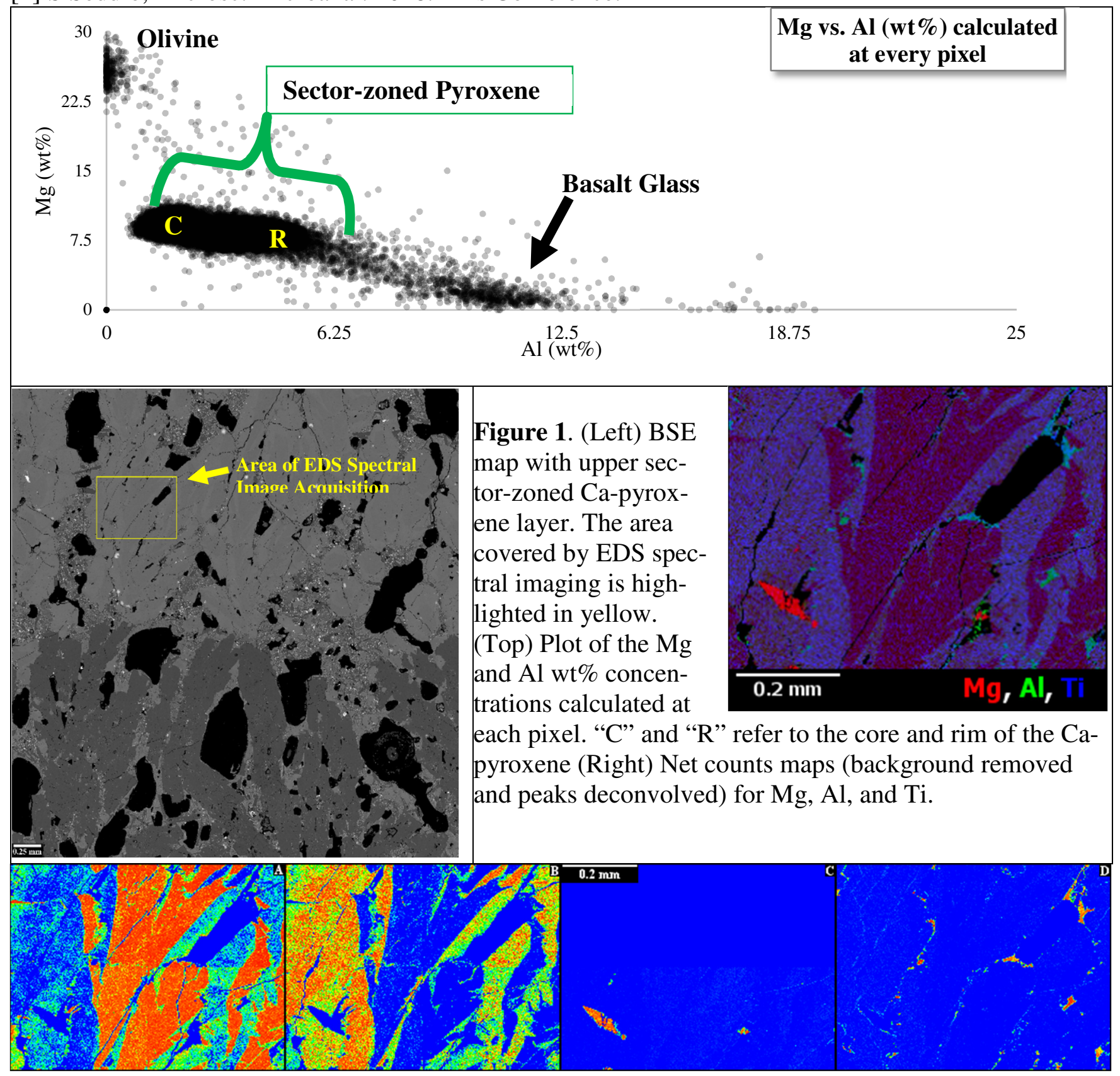

Figure 2. Component maps calculated from EDS spectral images using multivariate statistical analysis. (A) represents the core zone of the Ca-pyroxene. (B) represents the rim zone of the Ca-pyroxene. (C) represents olivine. (D) represents basalt glass. 